\title{
Simple system using natural mineral water for high-throughput phenotyping of Arabidopsis thaliana seedlings in liquid culture
}

This article was published in the following Dove Press journal:

International Journal of High Throughput Screening

2 March 2013

Number of times this article has been viewed

\author{
Abdelilah Benamar' \\ Antoine Pierart ${ }^{\prime}$ \\ Volker Baecker ${ }^{2}$ \\ Marie-Hélène Avelange- \\ Macherel $^{3}$ \\ Aurélia Rolland' \\ Sabine Gaudichon ${ }^{4}$ \\ Lodovico di Gioia ${ }^{4}$ \\ David Macherel' \\ 'Université d'Angers, Lunam \\ Université, Angers, ${ }^{2}$ MRI-Montpellier \\ RIO Imaging, Montpellier, \\ ${ }^{3}$ Agrocampus Ouest, Angers, ${ }^{4}$ Danone \\ Research, Palaiseau Cedex, France
}

Background: Phenotyping for plant stress tolerance is an essential component of many research projects. Because screening of high numbers of plants and multiple conditions remains technically challenging and costly, there is a need for simple methods to carry out large-scale phenotyping in the laboratory.

Methods: We developed a method for phenotyping the germination and seedling growth of Arabidopsis (Arabidopsis thaliana) Col-0 in liquid culture. Culture was performed under rotary shaking in multiwell plates, using Evian natural mineral water as a medium. Nondestructive and accurate quantification of green pixels by digital image analysis allowed monitoring of growth.

Results: The composition of the water prevented excessive root elongation growth that would otherwise lead to clumping of seedlings observed when classic nutrient-rich medium or deionized water is used. There was no need to maintain the cultures under aseptic conditions, and seedlings, which are photosynthetic, remained healthy for several weeks. Several proof-of-concept experiments demonstrated the usefulness of the approach for environmental stress phenotyping. Conclusion: The system described here is easy to set up, cost-effective, and enables a single researcher to screen large numbers of lines under various conditions. The simplicity of the method clearly makes it amenable to high-throughput phenotyping using robotics.

Keywords: phenotyping, phenomics, stress, seed, seedling, image analysis

\section{Introduction}

Arabidopsis (Arabidopsis thaliana (L.) Heynh) is a modest weed that, over the last 30 years, has gained the status of a premiere model in plant biology because of its genetic usefulness, ease of transformation, and small genome. ${ }^{1}$ Therefore, it is the most studied plant species, as testified by several thousands of publications each year. Most research projects on Arabidopsis involve, at some stage, an evaluation of the interactions between genotype, phenotype, and environmental conditions. Typically, this will occur at the beginning of a project to examine the response to abiotic or biotic stress, or at a later stage when the project addresses the functional validation of genes by reverse genetics. We will refer to phenotyping as the processes by which researchers evaluate the impact of environment and/or genotype on plant shape, growth, and physiology. It is straightforward to phenotype a collection of genotypes grown under the controlled conditions generally used in the laboratory by simply comparing growth (eg, biomass, morphological features, flowering time) and physiological parameters (eg, photosynthesis). However, many genotypes will only modify their phenotypes under particular conditions, which differ from controlled ones, sometimes within a narrow range of environmental parameters.
Correspondence: David Macherel UMR I 345 IRHS, Bat ARES, I6 Bd Lavoisier, F-49045 Angers, France Tel +3302 4I22 553I

Fax +330241225549

Email david.macherel@univ-angers.fr 
Therefore, it is important to be able to phenotype plants under various environmental conditions, which can become complex and technically demanding. If we consider temperature, which is a major physical parameter of the environment, studying the response of a collection of genotypes to simple heat shock at different temperatures is not trivial. The same observations apply for cold treatments, which are generally further limited by access to costly low temperature incubators, available cold rooms often being the only solution. In the case of other abiotic stresses (eg, light, saline, osmotic, dehydration, freezing, chemicals) similar or different difficulties (eg, interactions with the growth substrate or medium) occur, which renders large scale environmental phenotyping challenging. Thus, there is an important need for methods facilitating plant phenotyping and data acquisition. By combining the fields of genomics, imaging, and robotics, highthroughput plant phenotyping has evolved into phenomics, ${ }^{2,3}$ and private and academic platforms for high-throughput phenotyping of large numbers of plants are being established in many countries (eg, The Plant Accelerator, http://www. plantaccelerator.org.au).

These state-of-the-art robotized platforms allow automatic acquisition and analysis of multiple parameters for plants growing in greenhouses or cabinets. The limitation for researchers lies in the limited range of environmental conditions that can be applied, and also the high cost incurred. Phenotyping plants at the seedling stage offers several advantages because large populations can be screened in a limited area, and being very young plants, seedlings are quite homogenous. For seedling phenotyping, seeds are usually dispersed onto solid Murashige and Skoog agar medium $^{4}$ in Petri dishes, and germinated after stratification (typically a few days in a refrigerator or cold room). The impact of environment on germination and/or seedling growth is monitored by incubating the plates under various conditions. By standing the plates vertically or at an angle, gravitropism is maintained, which allows measurements of hypocotyl and root elongation, a system easily amenable to image analysis. ${ }^{5}$

All these systems are very useful, but require skilled manipulation for seed distribution and seed surface sterilization, and often suffer from contamination by micro-organisms. Water can be used as a simple medium, but this does not solve the problem of seed distribution, and agar gel remains a complex substrate suspected of modifying the bioavailability of xenobiotic compounds and is still prone to contamination. An alternative is to grow seedlings in shaking liquid cultures, generally in Murashige and Skoog medium. Such liquid cultures are more amenable to controlled temperature in the case of thermal stress, while another advantage is the constant and homogenous exposure of seedlings to any xenobiotics. ${ }^{6,7}$ The liquid culture technique also allows production of large amounts of seedlings, which is necessary for isolation of organelles. ${ }^{8}$ The main drawback is a high risk of fungal and bacterial contamination, which hampers the acquisition of sequential data.

Here, we have devised a very simple and robust phenotyping method for Arabidopsis Col-0 seedlings, including a noninvasive quantification of seedlings by image analysis. Seeds are germinated and grown in multiwell plates, using Evian natural mineral water as a medium, which prevents clumping and does not require maintenance of aseptic conditions. The usefulness and potential applications of the system, including adaptation to other species, are illustrated by several proof-of-concept experiments.

\section{Materials and methods Plant materials and growth conditions}

For seed production, Arabidopsis thaliana (Ecotypes Col-0, Ler, Sha, Bay-0, Cvi), Craterostigma plantagineum, and Nicotiana tabacum were cultured on soil in a growth room (temperature $23^{\circ} \mathrm{C}, 75 \%$ relative humidity, 16 hours of light, and a light intensity of $80-100 \mu \mathrm{mol} / \mathrm{m}^{2} /$ second).

\section{Liquid cultures}

Standardized amounts of dry seeds were inoculated in the wells of 6-well or 12-well plates (CytoOne Starlab, Bagnieux, France) containing $3 \mathrm{~mL}$ or $6 \mathrm{~mL}$ deionized water or Evian natural mineral water, using the volumetric method detailed in Figure S1. Multiwell plates with their lids were then incubated in the growth room (under the same conditions as above), on a rotary shaker, at $130 \mathrm{rpm}$. Plates were not stacked in order to ensure comparable illumination.

\section{Image analysis}

Digital images of the multiwell plates without their lids were acquired with a Nikon D5000 (Nikon France SAS, Champigny sur Marne, France) single lens reflex camera equipped with a $60 \mathrm{~mm}$ Nikkor microlens, using a stand (additional Figure S1). JPEG images were then analyzed in batch mode with ImageJ software (http://rsb.info.nih.gov/ij/), using a dedicated macro available as a supplementary material (Supplementary File 1). The macro was built to extract and quantify green pixels in each individual well. Pixel numbers are automatically reported in an Excel data file, and a control image for each well, containing the detected green 
pixels, is saved into a directory. This allows for the data to be examined by eye.

\section{Biomass, chlorophyll, and root length measurements}

To measure biomass, the seedling suspension was filtered with a $0.45 \mu \mathrm{m}$ nylon membrane (Millipore France, Molsheim, France) using a water-driven vacuum pump aspirator. Seedlings were scraped from the surface with a flat spatula and weighed to determine their fresh mass. Samples were then stored at $-20^{\circ} \mathrm{C}$. For chlorophyll assays, frozen samples were thawed on ice, and placed in glass tubes $(35 \times 12 \mathrm{~mm})$ containing $2 \mathrm{~mL}$ of dimethylformamide. After 24 hours of incubation at $4{ }^{\circ} \mathrm{C}$ in the dark, the tubes were vortexed, and $500 \mu \mathrm{L}$ was removed and centrifuged $(13,000 \mathrm{~g}, 5$ minutes $)$ to remove any fragments. $\mathrm{A}_{647}$ and $\mathrm{A}_{664}$ of the extract were measured by spectrophotometry (Fluostar Omega, BMG LabTech GmbH, Ortenberg, Germany). Total chlorophyll (Chlorophyll = 7.04 $\mathrm{A}_{664}+20.27 \mathrm{~A}_{647}$, in $\mu \mathrm{g} / \mathrm{mL}$ ) was calculated according to Moran. ${ }^{9}$ For root length measurements, 10-20 individual seedlings were laid on a black nylon filter (Schleicher and Schüell, MicroScience GmBH, Dassel, Germany) and photographed, and the length of the primary root was measured using ImageJ software.

\section{Natural and synthetic mineral waters}

Deionized water with an average conductivity of $30 \mu \mathrm{S} / \mathrm{cm}$ was obtained using a Milli-Q water system (Millipore France). The Evian natural mineral water outflows near the city of Evian (Haute-Savoie, France) in the French Alps from a well protected quaternary glacial sedimentary aquifer. During the mean transfer time within the aquifer of more than 15 years, it acquires its specific and constant composition (dry residue at $180^{\circ} \mathrm{C}=309 \mathrm{mg} / \mathrm{L}$ ) and neutral $\mathrm{pH}$ of 7.2. Evian natural mineral water was bought from commercial stores as $250 \mathrm{~mL}$ polyethylene terephthalate bottles stored in the dark. All experiments were performed with freshly opened bottles. Other brands of natural mineral water with various mineralization (Volvic, $130 \mathrm{mg} / \mathrm{L}$; Vittel, $844 \mathrm{mg} / \mathrm{L}$; Contrex, $2078 \mathrm{mg} / \mathrm{L}$; Hepar $2513 \mathrm{mg} / \mathrm{L}$ ) were bought from commercial stores. The various mineral-free waters were prepared by dissolving analytical grade mineral salts in Milli-Q water (Table 1). The water conductivity was measured with a Metrohm 712 conductivity meter (Metrohm, Villebonsur-Yvette, France) and the $\mathrm{pH}$ by potentiometric titration with a calomel-KCl electrode (736 GP Titrino, Metrohm). Calcium, magnesium, sodium, and potassium concentrations were verified using plasma emission spectroscopy with a
Spectroflame D (Spectro Analytical Instruments GmBH, Kleve, Germany). Chloride, sulfate, and nitrate composition was verified using ion chromatography according to Standard Methods 4110, using Dionex devices (Thermo Scientific, Sunnyvale, CA, USA). Bicarbonate content was measured by potentiometric titration using the 736 GP Titrino.

\section{Conductivity measurements}

Conductivity of the seedling culture medium was measured by carefully removing the liquid by aspiration, and after filtration on Miracloth (Merck KGaA, Darmstadt, Germany), conductivity was measured with a PC $5000 \mathrm{~L}$ meter equipped with a pHenomenal ${ }^{\circledR}$ CO11 electrode (WWR France, Fontenay-sous-Bois, France).

\section{Oxygen measurements}

The oxygen concentration of the medium (Evian natural mineral water) during culture was measured directly using an oxygen-sensitive microsensor ( $30 \mu \mathrm{m}$ tip) connected to a fiber optic oxygen transmitter (Microx TX3, Presens, Neuburg, Germany). Respiration and photosynthesis of 7-dayold seedlings were monitored using an Oxygraph oxygen electrode system (Hansatech, King's Lynn, UK) at $25^{\circ} \mathrm{C}$. Seedlings were collected by filtration on Miracloth (Merck $\mathrm{KGaA}$, Darmstadt, Germany) and $30 \mathrm{mg}$ fresh weight was introduced into the electrode chamber containing $1.5 \mathrm{~mL}$ of $10 \mathrm{mM}$ sucrose, $4 \mathrm{mM} \mathrm{KH}_{2} \mathrm{PO}_{4}$, $\mathrm{pH}$ 6.8. The system was operated at maximum stirring speed, and respiratory oxygen consumption was measured in the dark, while photosynthetic oxygen evolution was measured upon illumination using an optic fiber illuminating system providing $700 \mu \mathrm{mol} / \mathrm{m}^{2} / \mathrm{sec}$.

\section{Results}

\section{Germination and early seedling growth is efficient in natural mineral water}

With the aim of establishing a system for stress phenotyping of Arabidopsis Col-0 seedlings in liquid culture, we first started with multiwell ( 6 or 12 ) plates filled with $1 \times$ or $0.5 \times$ Murashige and Skoog medium ${ }^{4}$ and inoculated with surface-sterilized seeds. The seeds germinated within 2 days, and seedling growth was very fast, leading to clumping of seedlings after a week (data not shown). However, and in spite of careful manipulation in aseptic conditions, a major drawback was the frequent contamination of individual wells with micro-organisms (fungi, bacteria). In particular, it was almost impossible to remove the lid during the culture period, as required for image acquisition or physiological analysis, without contaminating the cultures. Further, seed surface ster- 
Table I Composition of Evian natural mineral water and synthetic waters

\begin{tabular}{|c|c|c|c|c|c|c|c|c|c|c|}
\hline $\mathrm{mg} / \mathrm{L}$ & $\mathrm{Ca}$ & $M g$ & $\mathrm{Na}$ & $\mathbf{K}$ & $\mathrm{HCO}_{3}$ & $\mathrm{Cl}$ & $\mathrm{SO}_{4}$ & $\mathrm{NO}_{3}$ & Total & $\mathrm{pH}$ \\
\hline EV & 80 & 26 & 6.5 & 1.0 & 360 & 6.8 & 12.6 & 3.7 & 497 & 7.2 \\
\hline Calcium-free & 0 & 27 & 56 & 57 & 349 & 7.0 & 13.0 & 3.8 & 512 & 7.4 \\
\hline Magnesium-free & 78 & 0 & 30 & 30 & 344 & 7.2 & 13.0 & 4.0 & 507 & 7.4 \\
\hline $\mathrm{Ca}-\mathrm{Mg}$ control & 78 & 25 & 53 & 55 & 369 & 134 & 12.0 & 3.4 & 730 & 7.5 \\
\hline Nitrate-free & 79 & 25 & 6.2 & I.I & 365 & 10.0 & 12.5 & 0 & 499 & 7.3 \\
\hline Sulfate-free & 82 & 26 & 6.5 & 2.4 & 371 & 19.0 & 0 & 3.5 & 511 & 7.3 \\
\hline
\end{tabular}

Notes: Mineral composition of water is indicated in $\mathrm{mg} / \mathrm{L}$ and $\mathrm{pH}$ in $\mathrm{pH}$ units. For the different mineral-free waters, compositions were balanced by using different salts. The $\mathrm{Ca}-\mathrm{Mg}$ free control was included to check the absence of influence of increased levels of $\mathrm{Na}$ and $\mathrm{K}$ in $\mathrm{Ca}$-free and $\mathrm{Mg}$-free waters.

Abbreviations: ABA, abscisic acid; DCMU, (3-(3,4-dichlorophenyl)-I,I-dimethylurea); DI, deionized water; EV, Evian natural mineral water; GA, gibberelic acid; PAC, paclobutrazol.

ilization was not reproducible. Because germination and early seedling growth require only water, we tried to use deionized water instead of Murashige and Skoog-based medium. When $200 \mathrm{mg}$ seeds were inoculated in $300 \mathrm{~mL}$ of deionized water in an Erlenmeyer flask placed on a shaker in the growth room, the seeds readily germinated and seedlings grew well, leading to clumping and formation of huge green rafts floating in the water (Figure 1). We postulated that clumping could result from the tangling of the long roots of individual seedlings under shaking conditions. Reasoning that the presence of

DI
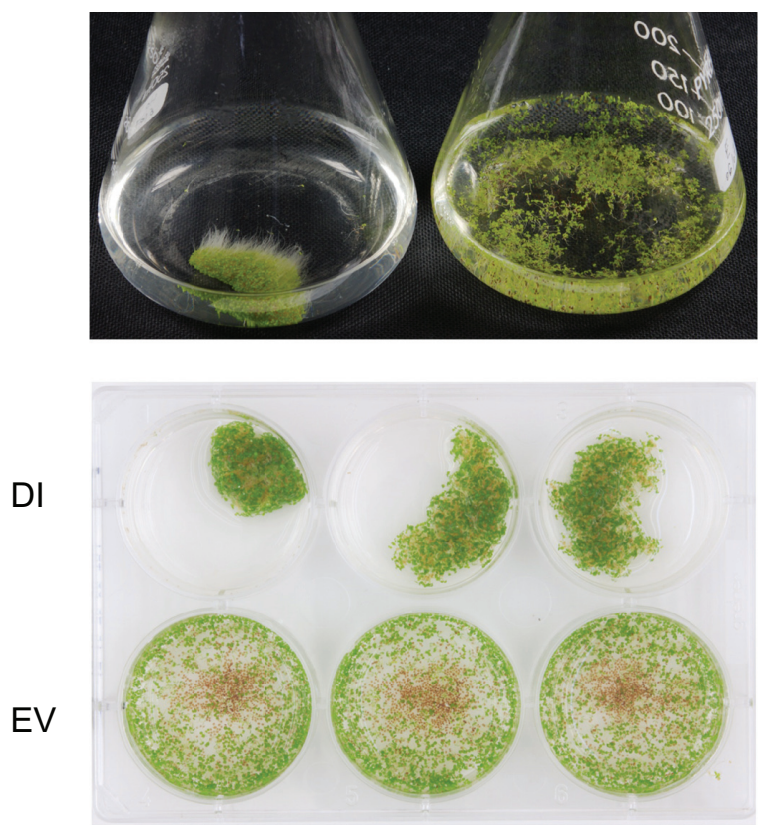

Figure I Effect of Evian natural mineral water on growth of Arabidopsis Col-0 seedlings in shaken liquid cultures.

Notes: The upper part shows Erlenmeyer flasks containing $100 \mathrm{~mL}$ of deionized water or Evian natural mineral water inoculated with $200 \mathrm{mg}$ dry seeds and photographed after 10 days of orbital shaking $(130 \mathrm{rpm}$ ) in a growth room ( 16 hours light at $23^{\circ} \mathrm{C}, 8$ hours dark at $18^{\circ} \mathrm{C}$ ). The lower part shows a multiwell plate after 7 days of culture under the same conditions, using $6 \mathrm{~mL}$ of either deionized or Evian natural mineral water inoculated with $20 \mathrm{mg}$ of Arabidopsis Col-0 dry seeds. Seedlings systematically aggregated in deionized water but remained dispersed in Evian natural mineral water.

Abbreviations: DI, deionized water; EV, Evian natural mineral water. some mineral elements in the solution could reduce root growth, and hence clumping, we performed the same experiment with Evian natural mineral water, which has a stable composition. As shown in Figure 1, seedlings readily germinated and grew well in Evian natural mineral water, without aggregation. Although neither the seeds nor the Erlenmeyer flasks, which were just capped with aluminum foil, were surface-sterilized, the seedlings could be kept in clean suspensions for several weeks, indicating that initial and subsequent maintenance of aseptic conditions was not required.

The length of the primary root of each individual seedling grown for 4 days was measured, revealing a large reduction in root length in Evian natural mineral water $(1.89 \pm 0.58 \mathrm{~mm})$ compared with deionized water $(6.62 \pm 1.16 \mathrm{~mm})$. The average length was thus reduced by a factor of 3.5 . We postulate that this reduction in root length is the main factor that prevents aggregation of Arabidopsis Col-0 seedlings during growth under shaking in the culture system. In deionized water, aggregation starts early as seedlings rapidly get tangled by their fine and long roots, and as the aggregates increase in size, more seedlings join, and often only one or a few rafts of tightly tangled seedlings can be found after a week of culture (Figure 1).

\section{Standardization of the system}

The system was further optimized for phenotyping Arabidopsis Col-0 germination and early seedling growth in multiwell plates under shaking using an orbital shaker placed in a controlled-environment growth room. With volumes of $6 \mathrm{~mL}$ (6-well) and $3 \mathrm{~mL}$ (12-well), orbital shaking (rotation $130 \mathrm{rpm}$ ) appeared ideal. As noticed before using Erlenmeyer flasks, the seedlings systematically aggregated in deionized water but not in Evian natural mineral water (Figure 1). Interestingly, seedling biomass production after 7 days was proportional to the initial amount of seeds $(11.7 \times$ initial mass of dry seeds) either in deionized or Evian natural mineral water. Moreover, seedlings could be maintained for 3 weeks in the system as detailed below. Condensation systematically 
occurred on top of the lids after a few days of culture. We did not find any efficient way to prevent this phenomenon, which was in fact inconsequential with regard to seedling growth or quality. However, if condensation were to be a concern, lids can be removed and changed at any time, or the big water droplets on the lid can be returned to the culture by a gentle tap. In order to achieve a reasonable density (ie, grown seedlings covering the well without excess), a ratio of $20 \mathrm{mg}$ of seeds for $6 \mathrm{~mL}$ medium is suitable. Given that weighing and manipulating milligram amounts of the tiny Arabidopsis seeds (which are electrostatic) is cumbersome, and may generate errors and increase the risk of seed dispersion in the laboratory, we designed a simple distributing tool (Figure S1) consisting of an aluminum or wooden plate in which cylindrical cups were drilled to different depths. Seeds are poured over the cup, overfilling the cavity. After gentle wiping of the surface with a flat metal spatula, a fixed volume of seeds remains in the cup, and is delivered into the well by flipping over the tool, leading to immediate seed dispersion in the medium (Figure S1). Calibration of cup filling by weighing indicates a very reproducible amount of seeds (eg, $20 \pm 0.5 \mathrm{mg}$ ). With this approach, a 6-well plate can be inoculated with well standardized amounts of seeds within a couple of minutes.

\section{Nondestructive measurement of growth by image analysis}

The usual approaches to estimate growth of plant materials (cells, tissues, seedlings) in a liquid system are measurement of object numbers, fresh/dry weight, and chlorophyll content. However, these methods, although quite accurate, are not well suited for high-throughput analysis because they are relatively complex to set up. In addition, being either damaging or destructive, they are not well suited to monitoring over time. Since the amount and density of green seedlings in the wells could be clearly discriminated by the naked eye, we decided to use image analysis to quantify precisely the amount of seedlings in the wells. A digital camera equipped with a fixed lens was mounted on a stand near to the rotary shaker, and a frame designed exactly to accommodate multiwell plates was attached to the stand's baseboard (Figure S1). With this system, multiwell plates can be removed from the shaker and photographed (without a lid), to acquire standardized digital images rapidly under fixed conditions. An automated procedure was devised, using the freely available ImageJ program and a dedicated macro, to extract the numbers of green pixels in each well automatically from the digital images. The macro allows saving of the filtered images of each well and inspection if required, while the number of green pixels per well is automatically exported to an Excel spreadsheet. The ImageJ macro is freely available as supplementary material (Supplementary File 1), and can be adapted to any plate format. The results shown in Figure 2 illustrate the accuracy of the whole process, with a relative coefficient of variation of $6.5 \%$ for green pixel values of six biological replicates. The detection is highly selective for green tissue, and we found that even a few germinated seedlings with green cotyledons in a single well were easily detected among several hundred nongerminated seeds or not yet green seedlings. The released brown integuments of germinated seeds sank to the bottom of the wells, and did not hamper image analysis (see Figure 2). By analyzing images of green card disks of known surface area placed in the plates, we determined that a plan area of $1 \mathrm{~mm}^{2}$ corresponded to 854 pixels in the images taken under our standardized conditions. Therefore, it was possible to relate measured pixel values to the projected surface of the green tissues of Arabidopsis seedlings. The high sensitivity of the

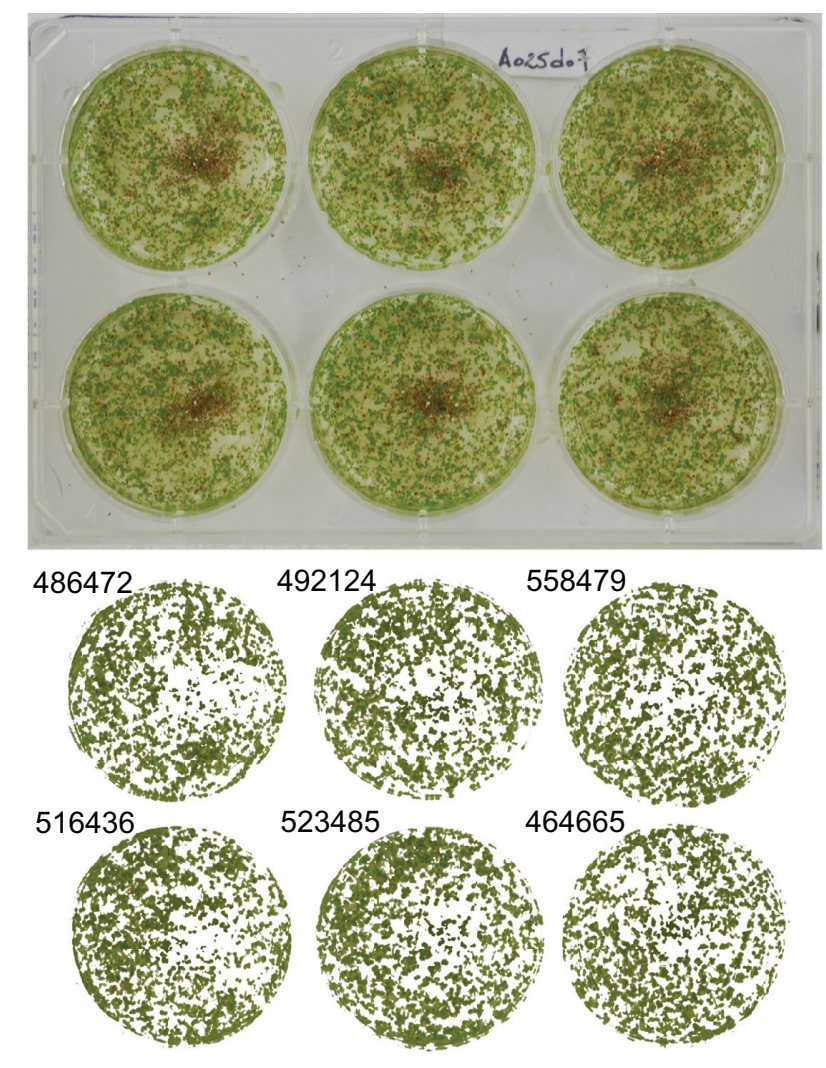

Figure 2 Measurement of seedling content in a multiwell plate by automated image analysis.

Notes: A multiwell plate was inoculated (each well with $20 \mathrm{mg}$ of dry Arabidopsis Col-0 seeds in $6 \mathrm{~mL}$ of Evian natural mineral water) and photographed after 7 days of culture. The image was automatically segmented and green pixels extracted and quantified using a dedicated ImageJ script (Supplementary File I). The corresponding green images of each well are shown below, together with the number of corresponding pixels. The average of the six well values is $506,943 \pm 33,002$. 
image analysis enabled establishment of an average green pixel value for a single seedling. Fixed numbers $(1,2,4$, $8,16,32)$ of 7 -day-old seedlings were placed in individual wells, in triplicate, and the plates were then subjected to image acquisition (three repeats, with shaking between each acquisition) and green pixel analysis. The results shown in Figure 3 demonstrate the sensitivity and reproducibility of the method, which clearly allows the detection of a single seedling in a well. By taking into account all the data (54 values corresponding to individual seedling pixels), a single 7-dayold seedling accounts for $183 \pm 5$ pixels.

\section{Kinetics of growth in deionized and Evian natural mineral water}

Using different assays (fresh weight, chlorophyll, green pixels), we compared the effect of deionized and Evian natural mineral water on seed germination and seedling growth during a 3-week culture in 6-well plates, with six replicates for each measurement. The results shown in Figure 4A indicate that seedlings germinate and grow very rapidly in both types of water during the first week with a more than 10-fold increase in biomass. Over the next 7 days the growth rate was much reduced, with an additional 7.5\% (deionized water) or $12 \%$ (Evian natural mineral water) increase in biomass by the end of the second week of culture. Noticeably, seedlings grown in liquid cultures (deionized or Evian natural mineral water) were unable to develop new leaves, indicating that their development is arrested at the cotyledon stage under such conditions. Nevertheless, they maintained their full potential for growth and development because, even after several

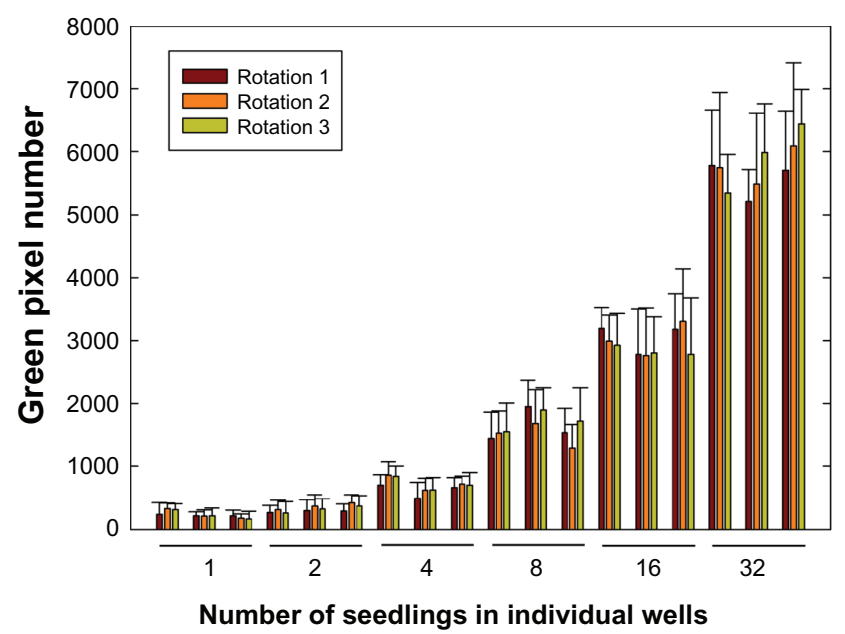

Figure 3 Quantification of seedling green pixel values.

Notes: A fixed number of 7-day-old seedlings grown in Evian natural mineral water was added to individual wells (in triplicate) of multiwell plates, and three successive images were acquired after shaking manually by rotation. The graph shows the average pixel values for each replicate and for the three different images (rotation I, 2, 3).
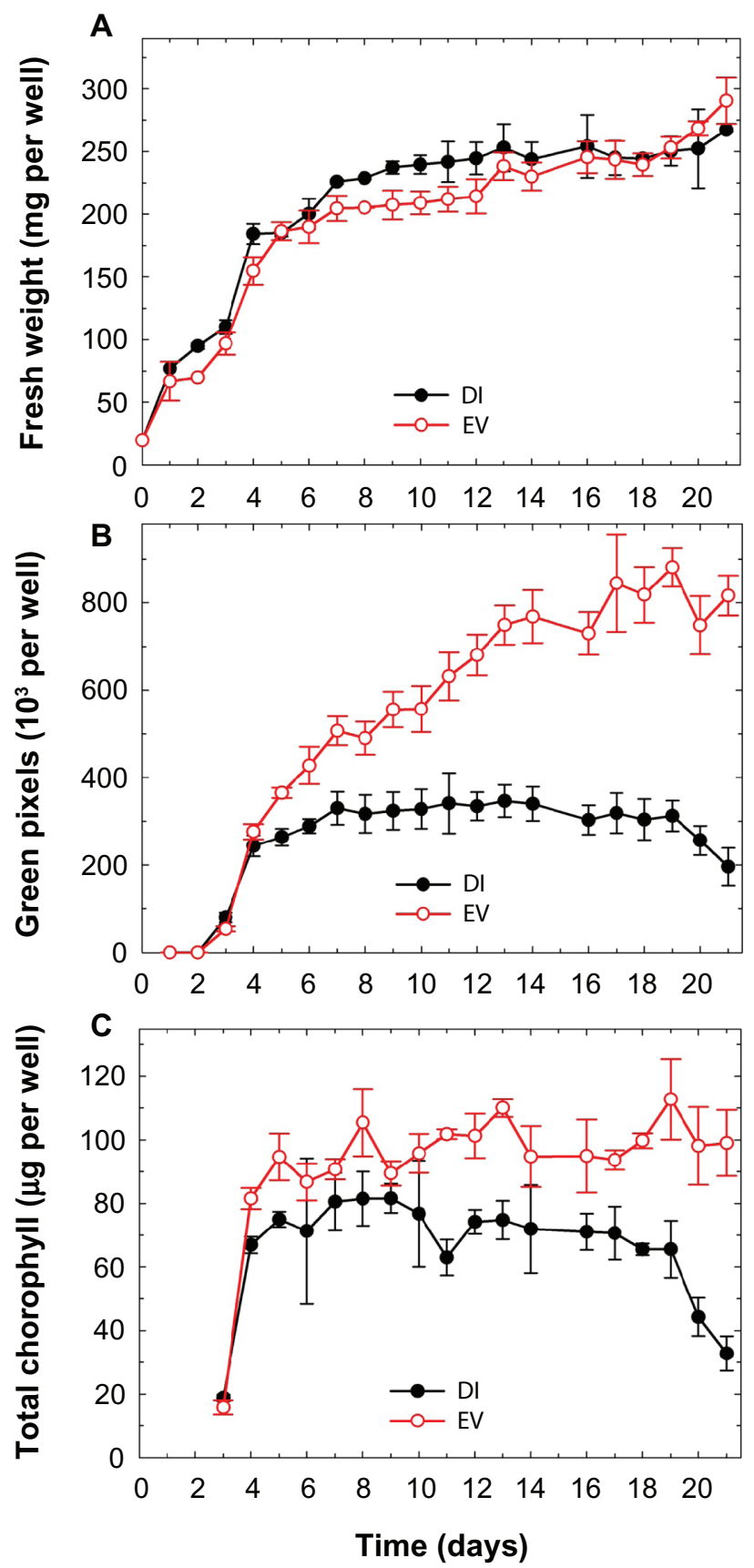

Figure 4 Changes in biomass, green pixel number, and chlorophyll during 3 weeks of culture.

Notes: Fresh weight $(\mathrm{A})$ was measured after collecting the well contents on a paper filter under vacuum. Green pixels (B) were quantified from digital images. Chlorophyll (C) was extracted from the same plant material with dimethylformamide and quantified in the clarified extract by spectrophotometry. The standard deviation (for triplicates) is indicated.

Abbreviations: DI, deionized water; EV, Evian natural mineral water.

weeks of liquid culture, seedlings transplanted to soil developed into healthy plants (results not shown). That seedlings aggregated in deionized water or remained dispersed in Evian natural mineral water had in fact no major effect on biomass production (Figure 4A). Green pixel quantification revealed 
that the imaging method is ideal for monitoring growth in Evian natural mineral water, but not in deionized water (Figure 4B). This is simply due to the masking of individual seedlings in the compact aggregates that form in deionized water, leading to a large underestimation of green surfaces. The correlation between green pixel accumulation and growth was confirmed by plotting fresh weight against green pixel quantification, using the data between 3 and 18 days of growth in Evian natural mineral water (Figure S2). Curve fitting showed that it is possible to convert green pixel data into biomass data, if required. Finally, the chlorophyll content shows a rapid increase following germination, followed by a plateau in the case of Evian natural mineral water and a slow decline in deionized water (Figure 4C). The plateau was reached earlier than in the case of biomass and green pixels, indicating that chlorophyll accumulation in cotyledons was almost achieved after 5-6 days, while cotyledon expansion still proceeds, as evidenced by green pixel analysis which does not take into account the green color density but only the green surface.

The chlorophyll content was always higher in the case of Evian natural mineral water and the increase in biomass is similar in Evian natural mineral water and deionized water, indicating that seedlings grown in Evian natural mineral water accumulate more chlorophyll than in deionized water. This observation is mainly due to the fact that seedlings grown in deionized water invest more in root biomass, at the expense of cotyledon growth. In deionized water, there is a sharp reduction in chlorophyll content and green pixels after 19 days (Figure 4C). This is due to the senescence of seedlings and is likely exacerbated by the lack of minerals in the culture.

The liquid culture system used here is obviously not equivalent to natural conditions for seedling growth because plants are submerged in the medium. In particular, one might wonder whether oxygen availability could be limiting. In order to control the oxygen status, we measured the oxygen concentration in the medium with a fluorometric oxygen sensing probe. The experiment was performed with 7-dayold seedlings, during both light and dark periods, to take into account possible photosynthetic oxygen evolution. The oxygen concentration was found to be almost constant, with a value around $260 \mu \mathrm{M}$, close to the oxygen saturation point of water at the temperature of the growth room $\left(20^{\circ} \mathrm{C}-23^{\circ} \mathrm{C}\right)$. This confirms that the medium was not hypoxic, ie, the oxygen concentration remained in equilibrium with the atmosphere, likely because of the high surface area to volume ratio of the medium and shaking. Neither photosynthetic oxygen evolution in the light nor the respiratory oxygen consumption of the seedlings overcame the rate of oxygen diffusion into the medium from the atmosphere, presumably because the mass of plant material was sufficiently low. As a whole, these results indicate that under experimental conditions, access to oxygen for metabolism is not a problem, and this correlates well with the rapid germination and seedling growth observed.

We next confirmed that seedlings grown in this liquid system were actively photosynthetic. One-week-old seedlings were transferred to the chamber of an oxygen electrode. Normally, organs or leaf tissues from plants grown in aerial conditions need to be vacuum infiltrated with the medium, to prevent floating and ensure gas diffusion between cells and the liquid phase where oxygen is monitored. Here this is not necessary because the seedlings grown in liquid are naturally infiltrated with the medium. The oxygraphic data indicate that seedlings are clearly photosynthetically active, with almost immediate oxygen evolution (values over $1.2 \mu \mathrm{mol} \mathrm{O}_{2} / \mathrm{min}$ per gram of fresh weight) upon illumination $\left(700 \mu \mathrm{mol} / \mathrm{m}^{2} / \mathrm{sec}\right)$ as shown in Figure S3. In the dark, respiratory oxygen consumption was in the range of $0.4-0.5 \mu \mathrm{mol} \mathrm{O} / \mathrm{min}$ per gram of fresh weight. Photosynthesis was completely inhibited by $10 \mu \mathrm{M}$ 3-(3,4-dichlorophenyl)-1,1-dimethylurea, an inhibitor of photosystem 2 , while addition of cyanide reduced respiration by more than $50 \%$ (Figure S3). The cyanide-resistant oxygen consumption is likely as a result of an alternative oxidase pathway because it is strongly affected by propyl gallate. For practical purposes, seedlings grown in Evian natural mineral water appear to be an excellent material for studying the impact of mutations or chemicals on photosynthesis and respiration because they offer a homogenous and intact material, which is amenable to measurement of gas exchange without infiltration. In addition, chemicals can diffuse toward cells directly within the liquid phase. Such seedlings are also a very handy material for teaching experiments about photosynthesis and respiration.

The conductivity of the culture medium was followed during 3 weeks of culture in deionized or Evian natural mineral water in order to characterize the global ionic concentration in the medium. As expected, the initial conductivity was much higher in Evian natural mineral water than in deionized water because of the ionic composition of Evian natural mineral water (Figure 5). In both cases, there was a transient increase at the beginning of the culture period (peak at day one), which is due to leakage of electrolytes from the seeds during imbibition. In deionized water, these compounds are rapidly 


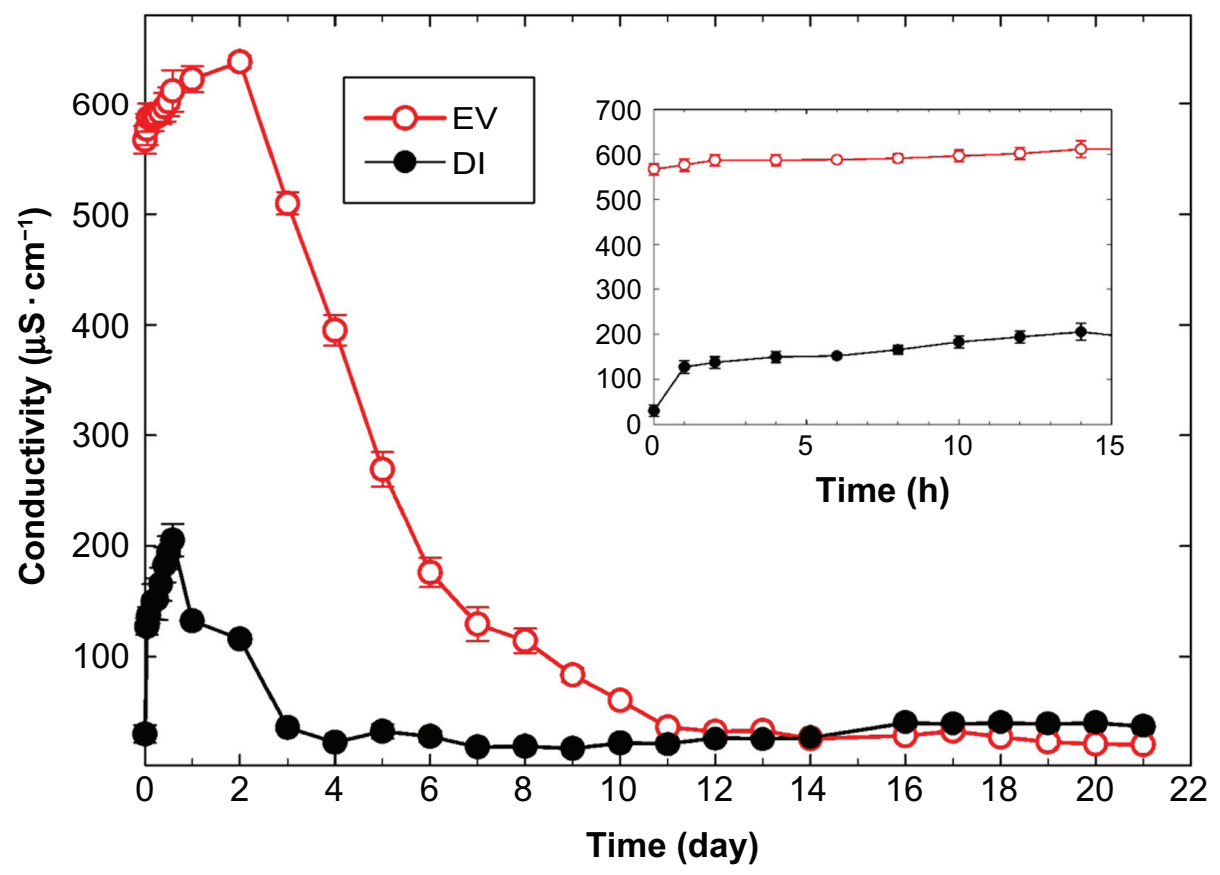

Figure 5 Change in conductivity of the medium during germination and seedling growth.

Notes: Multiwell plates were inoculated (each well with $20 \mathrm{mg}$ of dry Arabidopsis Col- 0 seeds in $6 \mathrm{~mL}$ of medium) with either deionized or Evian natural mineral water, and conductivity was measured at different time points by temporarily withdrawing $4 \mathrm{~mL}$ of the medium. The standard deviation (for triplicates) is indicated. The insert shows details of the early recording.

Abbreviations: DI, deionized water; EV, Evian natural mineral water.

taken up by the germinating seeds, and conductivity remains very low because the seedlings are have a high requirement for minerals. In Evian natural mineral water, after the first small increase, conductivity steadily decreased for up to 6 days, and then the rate decreased by half until all minerals are absorbed by the seedlings (around 11 days). Overall, these experiments clearly illustrate that Evian natural mineral water is a valuable resource for the young seedlings, contributing to their vigor in the liquid culture system.

\section{Impact of water composition on early development in liquid culture}

The dramatic difference in root elongation and clumping between seedlings grown in deionized water and Evian natural mineral water led us to investigate whether a specific component of natural mineral water could be responsible for the short root phenotype. Based on the composition of Evian natural mineral water, calcium-free, nitrate-free, sulfate-free, and magnesium-free waters were prepared, and chemically balanced to maintain $\mathrm{pH}$ and conductivity as close as possible to the original. In order to exclude any effect of an increased concentration of sodium and potassium in calcium-free and magnesium-free waters, a calcium-magnesium control water was included (see Materials and methods section). Root length measured after 4 days of culture and seedling growth after one week was similar to that in Evian natural mineral water for all solutions (Figure S4). No obvious difference from Evian natural mineral water could be demonstrated for growth or aggregation in nitrate-free, sulfate-free, and magnesium-free waters or calcium-magnesium control water. In the case of calcium-free water, occasional wells (1/6) displayed seedling aggregation, and seedlings appeared slightly paler (data not shown). We also examined the effect of individual mineral salts added alone to deionized water, at concentrations approximating those in Evian natural mineral water, on the tendency of seedlings to aggregate. $\mathrm{Ca}\left(\mathrm{HCO}_{3}\right)_{2}$, $\mathrm{CaCl}_{2}$, or $\mathrm{CaSO}_{4}$ did not prevent aggregation of seedlings, while in the case of magnesium salts $\left(\mathrm{MgSO}_{4}\right.$ or $\left.\mathrm{MgCl}_{2}\right)$, the proportion of wells displaying aggregation was reduced to $50 \%$. However, seedlings grown in magnesium salts did not display shorter roots when measured at 4 or 7 days (data not shown). Therefore, it is not possible to correlate this lesser tendency to aggregate in magnesium salts with root growth. Several brands of mineral waters with various compositions and a higher or lower dry residue than Evian natural mineral water were assayed, but none could systematically prevent aggregation of Arabidopsis Col-0 in the liquid culture system (Figure S5).

In summary, these experiments illustrate the complexity of the response of seedling development to the mineral composition of water, and indicate that it is not a single compound or element but rather the unique overall combination 
of elements and concentrations in the solution that favors vigorous growth without aggregation of seedlings in liquid culture. To demonstrate the interest in and potential of this system for Arabidopsis Col-0 phenotyping in various conditions, we have conducted several proof-of-concept experiments.

\section{Hormonal control of germination}

The phytohormones, abscisic acid and gibberelic acid promote seed dormancy and germination, respectively, and thus the balance between gibberelic acid and abscisic acid is a major controller of seed germination. ${ }^{10}$ We have verified that abscisic acid strongly inhibited germination in the $\mu \mathrm{M}$ range, with total blockage of germination at $0.5 \mu \mathrm{M}$ (Figure 6). Similarly, paclobutrazol, which is an inhibitor of biosynthesis of gibberelic acid, strongly inhibited germination at a similar range of concentrations (Figure 6). This shows that the system is well suited to analyze the effect of compounds/conditions interfering with seed dormancy and germination.

\section{Chemicals}

Menadione is a quinone known to interfere with electron transfer in mitochondria, resulting in the production of reactive oxygen species by redox cycling, thus triggering oxidative stress. ${ }^{11}$ The toxicity of menadione towards Arabidopsis Col-0 seedlings was easily detected with the system (Figure 7). Concentrations over $10 \mu \mathrm{M}$ resulted in a strong reduction of growth as quantified by green pixel analysis. Such concentrations also delayed germination

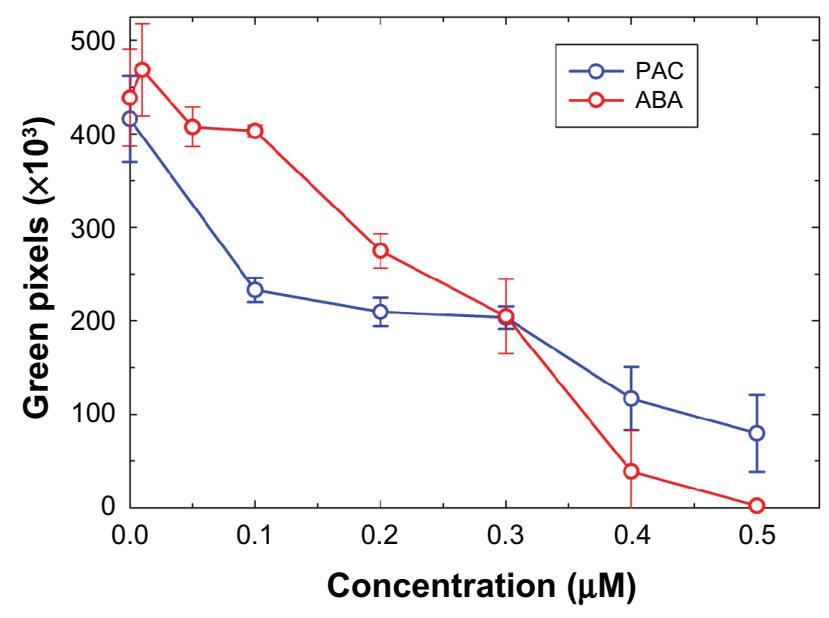

Figure 6 Effect of abscisic acid and paclobutrazol on germination and early growth of Arabidopsis seedlings.

Notes: Seeds were inoculated in the wells of multiwell plates $(20 \mathrm{mg}$ of dry Arabidopsis Col-0 seeds in $6 \mathrm{~mL}$ of Evian natural mineral water) with various concentrations of abscisic acid or paclobutrazol. Green pixels were quantified after 7 days of culture. The experiment was performed in triplicate, and the standard deviation is indicated on the graph.

Abbreviations: ABA, abscisic acid; GA, gibberelic acid; PAC, paclobutrazol.

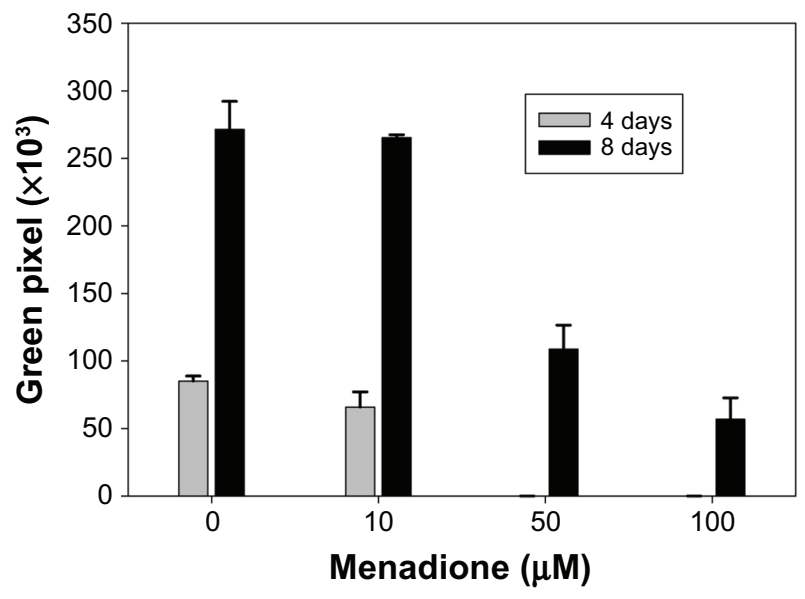

Figure 7 Effect of menadione on germination and growth of Arabidopsis seedlings. Notes: Seeds were inoculated in the wells of multiwell plates $(20 \mathrm{mg}$ of dry Arabidopsis Col-0 seeds in $6 \mathrm{~mL}$ of Evian natural mineral water) with various concentrations of menadione. Green pixels were quantified after 4 and 8 days. The experiment was performed in triplicate, and the standard deviation is indicated on the graph.

because no seedlings were detected after 4 days. When menadione $50-100 \mu \mathrm{M}$ was added at day 4 , ie, when seeds had already germinated, growth was severely compromised, with no further increase in green pixels 4 days later (result not shown). This indicates that menadione at concentrations over $10 \mu \mathrm{M}$ has a moderate impact on seed germination but a strong effect on seedling growth. Overall, the system allows easy monitoring of the toxicity of different chemicals, and is especially suitable for high-throughput preliminary screening of multiple concentrations of various compounds expected to interfere with plant metabolism and growth.

\section{Temperature tolerance}

The system was used to examine the tolerance of Arabidopsis Col-0 to extreme temperatures. Freezing stress (one night at $-18^{\circ} \mathrm{C}$ ) or heat shock $\left(90\right.$ minutes at $42^{\circ} \mathrm{C}$ or $48^{\circ} \mathrm{C}$ ) was applied to the seedlings grown in Evian natural mineral water for one (nongerminated) or 4 days (seedlings), which were then placed back in normal conditions. Their survival was estimated 10 days after treatment by quantifying green pixels. Clearly, freezing induced severe effects because only a few seedlings survived the treatment in all cases (Figure 8). Although we have not explored further cold and freezing tolerance (and acclimation), the system could be easily used to analyze the effects of shorter exposure to freezing (or a higher freezing temperature), or prolonged incubation at $0^{\circ} \mathrm{C}$. Only a few seedlings survived heat shock at $48^{\circ} \mathrm{C}$ (Figure 8), which is a lethal temperature for most plants without previous acclimation. Interestingly, the heat shock at $42^{\circ} \mathrm{C}$ almost killed all the 4-day-old seedlings, but was much better 


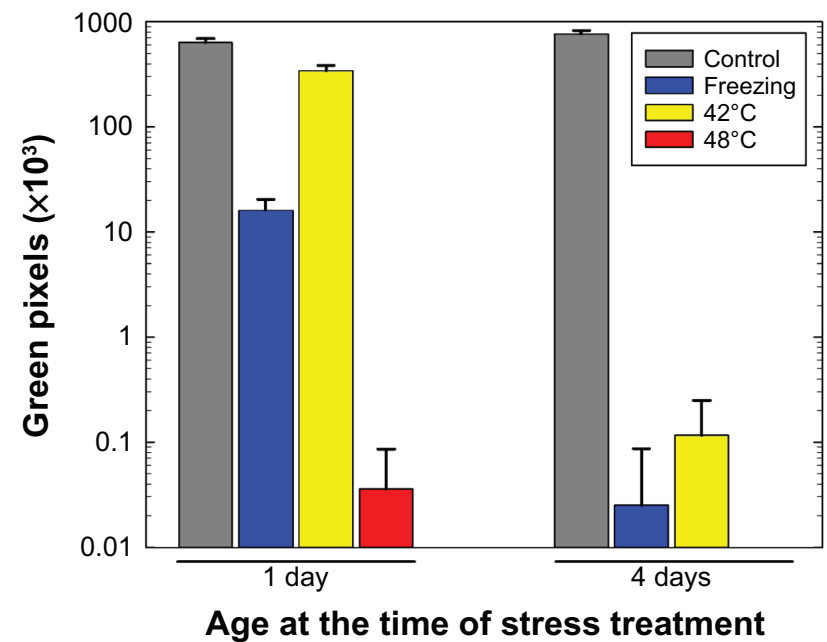

Figure 8 Effect of freezing and heat shock on the germination and growth of Arabidopsis seedlings.

Notes: Seeds were inoculated in the wells of multiwell plates $(20 \mathrm{mg}$ of dry Arabidopsis Col-0 seeds in $6 \mathrm{~mL}$ of deionized or Evian natural mineral water). At the times indicated, the plates were subjected to freezing overnight $\left(-18^{\circ} \mathrm{C}\right)$ or 90 minutes of heat shock at $42^{\circ} \mathrm{C}$ or $48^{\circ} \mathrm{C}$, using incubation of sealed plates in a waterbath. After the stress treatments, seedlings were grown for 10 days under control conditions, and survival was quantified by green pixel analysis. The pixel data are shown using a logarithmic scale.

tolerated by nongerminated seeds that had imbibed for only one day (Figure 8), because the value of green pixels measured 10 days after treatment was half that of the untreated seedlings (shown in Figure 4B). This indicates that nongerminated seeds are still benefiting from stress protection mechanisms (eg, heat shock proteins and proteins abundant during late embryogenesis) associated with tolerance of desiccation. ${ }^{12}$ These simple experiments show that the system is very convenient for the study of the effects of temperature on Arabidopsis seedlings. Large numbers of homogeneous seedlings can be subjected to a variety of temperature treatments, which can be precisely controlled by incubating the multiwell plates in water baths. Such flexibility in the control of temperature is especially important when taking into account how plant responses are dramatically influenced by different temperature regimes. ${ }^{13}$ In addition, the system is well suited to isolate and characterize individual plants that show an unusual tolerance (eg, survivors of $48^{\circ} \mathrm{C}$ heat shock).

\section{Phenotyping other accessions and species}

All parts of the procedure were developed with the ecotype Col-0 which is the most studied accession of Arabidopsis. When grown in Evian natural mineral water, the other widely used ecotype Ler did not show aggregation, while the ecotypes Bay-0, Ws, and Sha systemically aggregated and the ecotype Cvi showed aggregation in one third of the wells. The method is thus not directly applicable to all
Arabidopsis ecotypes. We have also assayed other species, focusing on small seeds because it is obvious that the system is not suitable for large seeds. It was found to work well with C. plantagineum (a resurrection plant) which behaved almost like Arabidopsis, with aggregation in deionized water but not in Evian natural mineral water (Figure S4). Interestingly, tobacco (N. tabacum) did not aggregate in either condition (Figure S6), but seedlings appeared greener in Evian natural mineral water.

\section{Discussion}

The system described here can greatly facilitate mediumthroughput to high-throughput phenotyping of seed germination and early seedling growth of Arabidopsis Col-0 and Ler. To our knowledge, the culture of Arabidopsis seedlings systematically uses rich medium (eg, $0.5 \times$ Murashige and Skoog with $2 \%$ sucrose), and requires aseptic conditions at every stage, including seed surface sterilization, as described elsewhere. ${ }^{8,14,15}$ In addition to a high occurrence of microbial contaminations, growth in a rich medium always leads to clumping of seedlings that, in turn, form larger rafts, which hamper measurements unless these are destructive. Interestingly, the addition of $0.1 \%$ agar was shown to prevent clumping of the Arabidopsis WS ecotype in liquid cultures performed in Murashige and Skoog medium, ${ }^{16}$ but this does not prevent contamination by micro-organisms. The major advantages of the method we describe here are that asepsis is not required at any stage and Arabidopsis Col-0 seedlings do not aggregate, which considerably facilitates the setting up and monitoring of large-scale experiments. The system has a low cost of consumables because multiwell plates can be reused and a $1.5 \mathrm{~L}$ bottle of Evian natural mineral water is sufficient for an experiment with 40 multiwell plates (6-well or 12-well plates). Moreover, the water has a very stable composition, and is commercially available in more than 140 countries, which allows standardization of experiments in different locations. From our results concerning the effect of water composition on the tendency of seedlings to aggregate, it is clear that other combinations of minerals, either in synthetic or in natural waters, might work as well. However, making salt solutions or using unreliable water sources increases the variability of the solution and risk of errors.

The simple and accurate inoculation procedure, as well as the fast and nondestructive quantification of germination/ growth by image analysis guarantees robust data with a minimum of effort. The variety of data we provide demonstrates the accuracy, sensitivity, and reproducibility of the system, which is especially suitable for stress phenotyping given that 
multiple conditions with replicates can be routinely tested. The medium can easily be changed to monitor the impact of noxious compounds and, for heat or cold stress, the temperature can be precisely controlled and stably maintained. Another advantage of the system is that seedlings can be easily recovered from the suspension and transplanted to soil. Therefore, the method should have applications for screening and isolating abiotic stress mutants.

In our laboratory, a single person can perform phenotyping experiments with several dozen multiwell plates and obtain statistically significant results in less than 2 weeks. The design of a multistage rotary shaker with integrated illumination would allow a great increase in the number of plates that could be screened relative to growing plants on agar on growth room shelves. Clearly, the system is amenable to high-throughput phenotyping using robotics. The fact that it does not require aseptic conditions and uses standard multiwell plates is a considerable advantage for such a prospect. The image analysis procedure could also, at least in theory, be implemented in order to monitor germination sensu stricto (ie, radical protrusion) by filtering and detecting the amount of white pixels corresponding to the emerging radicle. However, the small size of the radicles and the density and distribution of seeds would prevent accurate analysis. Moreover, given that Arabidopsis germination is rapid and occurs at 15-45 hours after imbibition, it would be necessary to acquire images at short time intervals (eg, every 2 hours) in order to build germination curves. An efficient system has already been developed for the high-throughput automatic scoring of Arabidopsis germination and curve fitting of cumulative data. ${ }^{17}$ In this approach, seeds are incubated on wet filter paper in trays with transparent lids, allowing image acquisition and scoring of germination based on color contrast between the radicle and seed coat. The system we describe here is not designed for the establishment of germination curves, but rather for the evaluation of seedling emergence and growth, and germination is in fact inferred from the appearance of green cotyledons.

A limitation lies in the fact that not all Arabidopsis ecotypes display the same properties, ie, lack of aggregation in Evian natural mineral water, which would preclude the quantification of seedling growth by green pixel analysis. However, it was pointed out that aggregation does not prevent growth (see Figure 4), and therefore the system can still be used with fresh weight quantification (which can be nondestructive) or chlorophyll content determination for stress tolerance screening of accessions or mutants in different backgrounds.
A drawback of the system is that plants do not normally grow in submerged conditions, and thus the physiological context of the system is not natural. This is illustrated by the fact that seedlings never develop true leaves under these conditions. However, we have ensured that the medium was not hypoxic, and that the mineral composition of Evian natural mineral water maintained seedlings which were photosynthetically active in a healthy state for weeks. We postulate that such a flooding situation can indeed be encountered in the field by the tiny Arabidopsis seeds (and likely other species), which are thus able to germinate and survive moderate submersion for several days as seedlings, waiting for ideal conditions (water drainage) to initiate their full development into vigorous plants. To analyze many different stress conditions or compounds using seedlings or plants grown in a more physiological situation would be much more difficult and more costly. There is a necessary tradeoff when using artificial conditions in phenotyping programs, and the first goal of such an approach is to uncover phenotypes in comparison with controls, to provide functional clues about the interactions between gene function and environmental constraints. Once phenotypes of interest are detected using this system, they can be studied in more physiological situations with reduced numbers of plants at various developmental stages.

\section{Conclusion}

We have developed a simple, economic, and robust approach that considerably facilitates the medium-scale to large-scale phenotyping of Arabidopsis Col-0 or Ler seed germination and seedling growth in liquid conditions. The principal advantage is the easy handling and analysis of the materials because asepsis is not required and seedlings do not clump. The method, which includes nondestructive measurements of growth by image analysis, is especially suitable for studying the impact of various abiotic stressors, and to screen compounds expected to impact on germination, metabolism, and early growth of seedlings.

\section{Acknowledgment}

We are grateful to David C Logan for careful proofreading of the manuscript.

\section{Disclosure}

The main medium used in this work, Evian natural mineral water, is the property of Danone. This study was partly supported by a grant from Danone Research, Daniel Carasso Research Centre, France. The authors report no other conflicts of interest in this work. 


\section{References}

1. Koornneef M, Meinke D. The development of Arabidopsis as a model plant. Plant. 2010;61:909-921.

2. Finkel E. With phenomics, plant scientists hope to shift breeding into overdrive. Science. 2009;325:380-381.

3. Furbank RT, Tester M. Phenomics - technologies to relieve the phenotyping bottleneck. Trends Plant Sci. 2011;16:635-644.

4. Murashige T, Skoog F. A revised medium for rapid growth and bio assays with tobacco tissue cultures. Physiol Plant. 1962;15:473-497.

5. MacGregor DR, Deak KI, Ingram PA, Malamy JE. Root system architecture in Arabidopsis grown in culture is regulated by sucrose uptake in the aerial tissues. Plant Cell. 2008;20:2643-2660.

6. Bae H, Herman E, Sicher R. Exogenous trehalose promotes non-structural carbohydrate accumulation and induces chemical detoxification and stress response proteins in Arabidopsis thaliana grown in liquid culture. Plant Sci. 2005;168:1293-1301.

7. Zouhar J, Hicks GR, Raikhel NV. Sorting inhibitors (sortins): chemical compounds to study vacuolar sorting in Arabidopsis. Proc Natl Acad Sci US A. 2004;101:9497-9501.

8. Escobar MA, Geisler DA, Rasmusson AG. Reorganization of the alternative pathways of the Arabidopsis respiratory chain by nitrogen supply: opposing effects of ammonium and nitrate. Plant J. 2006;45:775-788.

9. Moran R. Formulae for determination of chlorophyllous pigments extracted with N,N-dimethylformamide. Plant Physiol. 1982;69: 1376-1381.
10. Yamaguchi S, Kamiya Y, Nambara E. Regulation of ABA and GA levels during seed development and germination in Arabidopsis. In: Bradford KJ, Nonogaki H, editors. Seed Development, Dormancy and Germination. Oxford, UK: Blackwell Publishing Ltd; 2007.

11. Frei B, Winterhalter KH, Richter C. Menadione-(2-methyl-1, 4-naphthoquinone) dependent enzymic redox cycling and calcium release by mitochondria. Biochemistry. 1986;25:4438-4443.

12. Hoekstra FA, Golovina EA, Buitink J. Mechanisms of plant desiccation tolerance. Trends Plant Sci. 2001;6:431-438.

13. Mittler R, Finka A, Goloubinoff P. How do plants feel the heat? Trends Biochem Sci. 2012;27:118-125.

14. Dai X, Hayashi Ki, Nozaki H, Cheng Y, Zhao Y. Genetic and chemical analyses of the action mechanisms of sirtinol in Arabidopsis. Proc Natl Acad Sci U S A. 2005;102:3129-3134.

15. Aghdasi M, Smeekens S, Schluepman H. Microarray analysis of gene expression patterns in Arabidopsis seedlings under trehalose, sucrose and sorbitol treatment. Int J Plant Prod. 2008;2:309-320.

16. Nichols KW, Heck GR, Fernandez DE. Simplified selection of transgenic Arabidopsis thaliana seed in liquid culture. Biotechniques. 1997;22:62-63.

17. Joosen RV, Kodde J, Willems LA, Ligterink W, van der Plas LH, Hilhorst HW. GERMINATOR: a software package for high-throughput scoring and curve fitting of Arabidopsis seed germination. Plant J. 2010;62:148-159. 


\section{Supplementary file I}

This zip file corresponds to the script package for automated quantification of green pixel analysis by ImageJ. Installation and getting started: copy the file macros/toolsets/ Arabidopsis_Seedlings_Tool.txt into the folder macros/ toolsets/of your ImageJ installation. Copy the .jar files from the folder plugins/IO into a subfolder (/IO) of the pluginsfolder of your ImageJ installation. Start ImageJ and select the

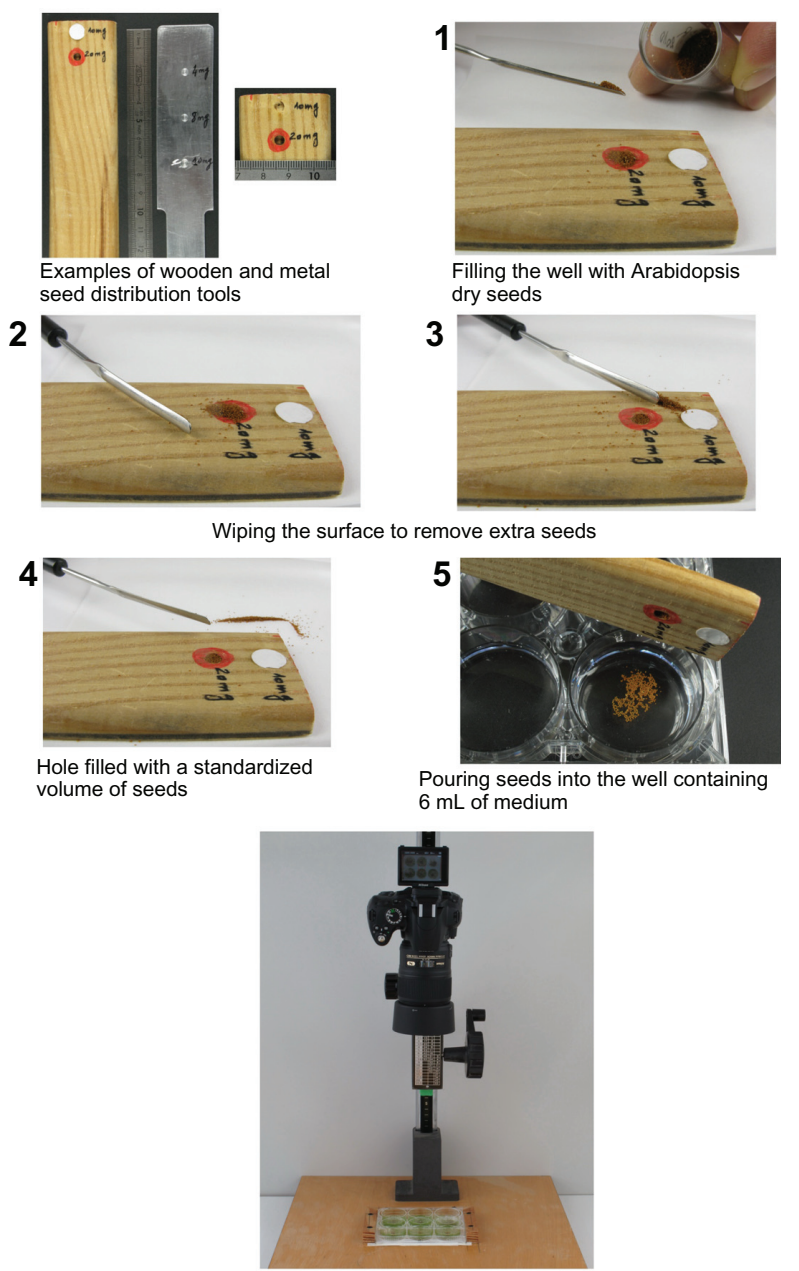

Figure SI Method for distributing equal amounts of Arabidopsis seeds into a multiwell plate and image acquisition setup.

Notes: A series of sequential images are shown to illustrate the distribution tools and key steps in the process. The experimental apparatus (stand, multiwell plate frame, and camera) used for rapid image acquisition of multiwell plates is also shown. Images can be acquired in JPEG format, using highest resolution (fine mode), preferentially in a manual mode to standardize exposure. The multiwell plate should be centered correctly and occupy the entire frame.
Arabidopsis_Seedlings_Tool from the toolset button $(>>)$ of the ImageJ-launcher window. Press the button with the image to open the help page of the tool in your web-browser. The tool is also available at http://dev.mri.cnrs.fr/wiki/ imagej-macros/Arabidopsis_Seedlings_Tool. The options menu of the ImageJ macro (right click on $r$ button) enables adaptation of the segmentation and filtering parameters to the user's images. 


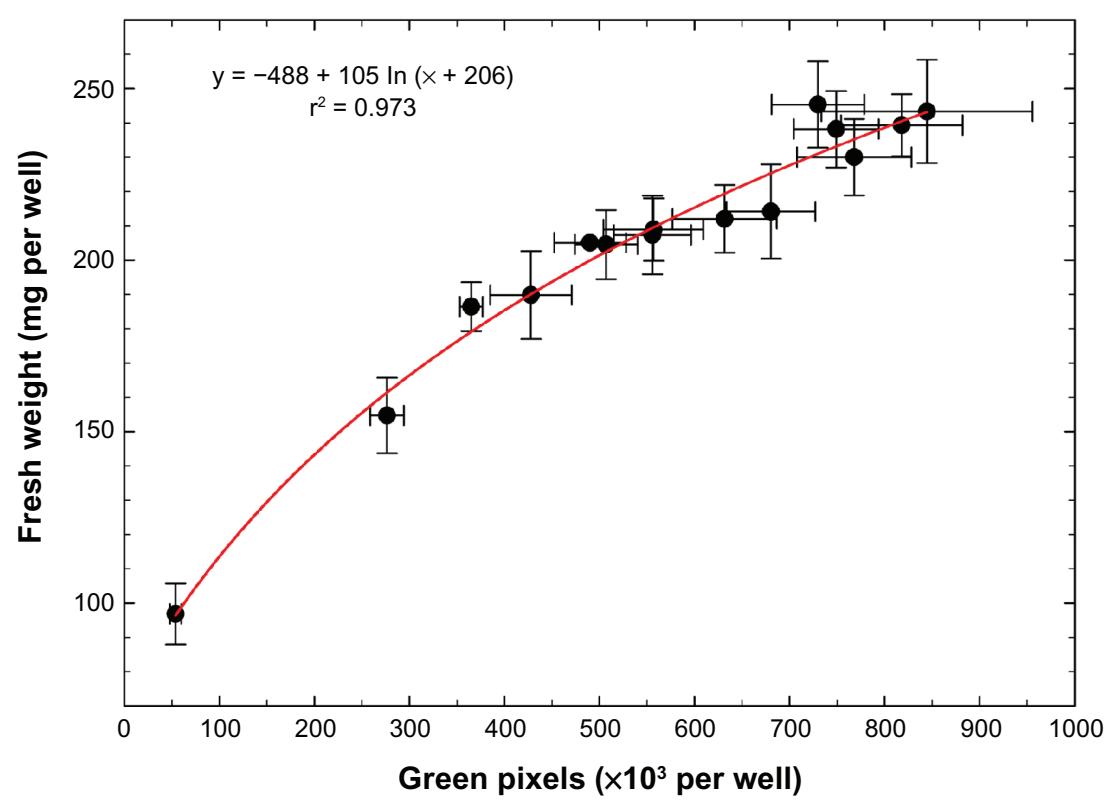

Figure S2 Correlation between green pixel accumulation and biomass.

Notes: Data corresponding to days 3-18 of culture in Evian natural mineral water in the kinetics experiment (Figure 4) were used to plot green pixel versus fresh weight. Curve fitting was performed using SigmaPlot version II.2.0.5 (Systat Software, San José, CA), with nonlinear regression and dynamic fitting to extract a logarithmic equation $\left(y=105 \ln (x+206)-488 ; r^{2}=0.973\right)$ that passed a Shapiro-Wilk normality test $(P=0.9043)$.

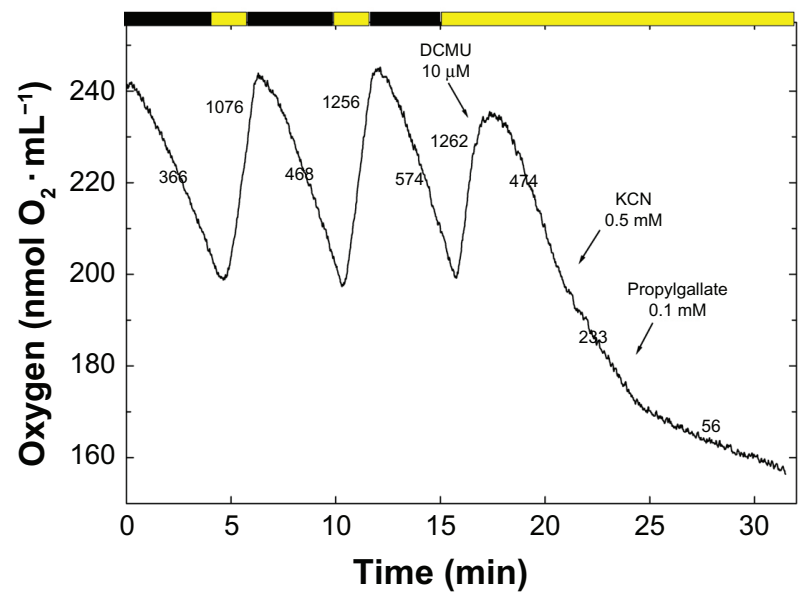

Figure S3 Photosynthesis and respiration of seedlings grown in liquid culture. Notes: Arabidopsis Col-0 seedlings (30 mg fresh weight) grown for one week in Evian natural mineral water were transferred to the chamber of an oxygen electrode containing $1.5 \mathrm{~mL}$ medium ( $10 \mathrm{mM}$ sucrose, $4 \mathrm{mM} \mathrm{K \textrm {H } _ { 2 }} \mathrm{PO}_{4}$, $\mathrm{pH} 6.8$ ). Oxygen concentration was measured at $25^{\circ} \mathrm{C}$ in the dark (black bar) or in the light (yellow bar, $700 \mu$ Einstein $/ \mathrm{m}^{2}$ per second). The addition of inhibitors is indicated by arrows. The numbers besides the trace correspond to oxygen consumption or evolution rates, in $\mathrm{nmol} \mathrm{O}_{2} / \mathrm{min}$ per gram fresh weight.

Abbreviation: DCMU, (3-(3,4-dichlorophenyl)-I,I-dimethylurea).
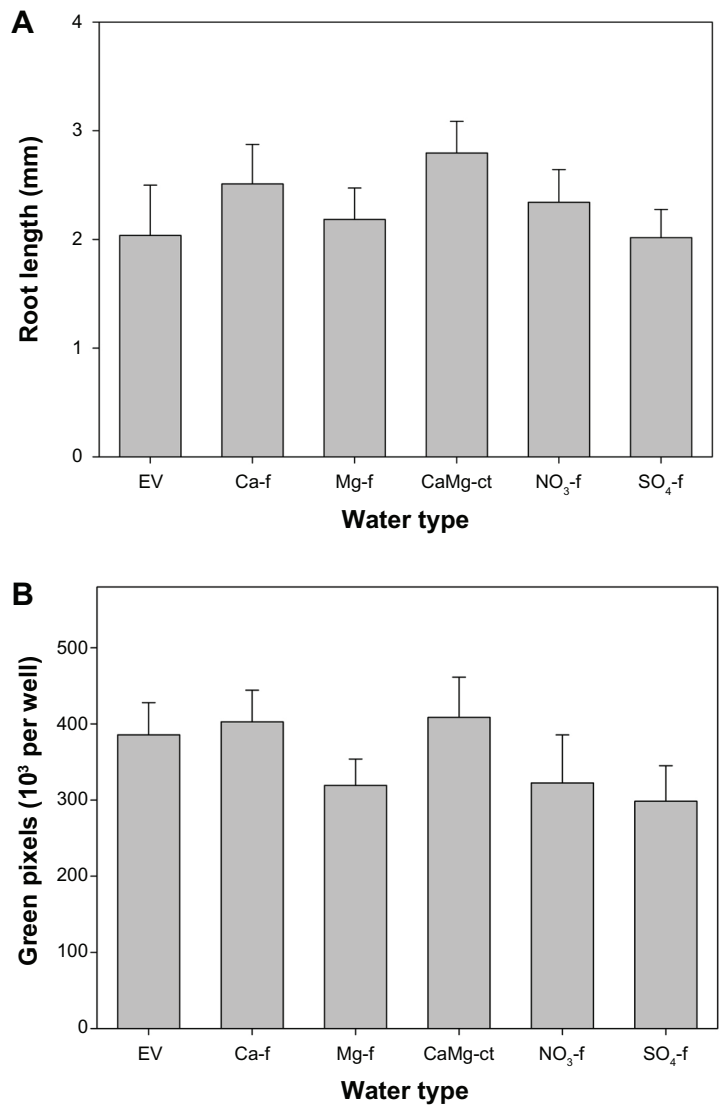

Figure S4 Assay of synthetic waters. Seedlings were grown in Evian natural mineral water or in synthetic waters with various compositions (see Table I). The upper graph (A) shows the average root length of seedlings measured after 4 days and the lower graph (B) shows the green pixel values measured after 7 days.

Abbreviations: Ca-f, calcium free, EV, Evian natural mineral water; Mg-f, magnesium free, $\mathrm{NO}_{3}-f$, nitrate free; $\mathrm{SO}_{4}-\mathrm{f}$, sulfate free; $\mathrm{CaMg}$-ct, calcium magnesium control. 
A

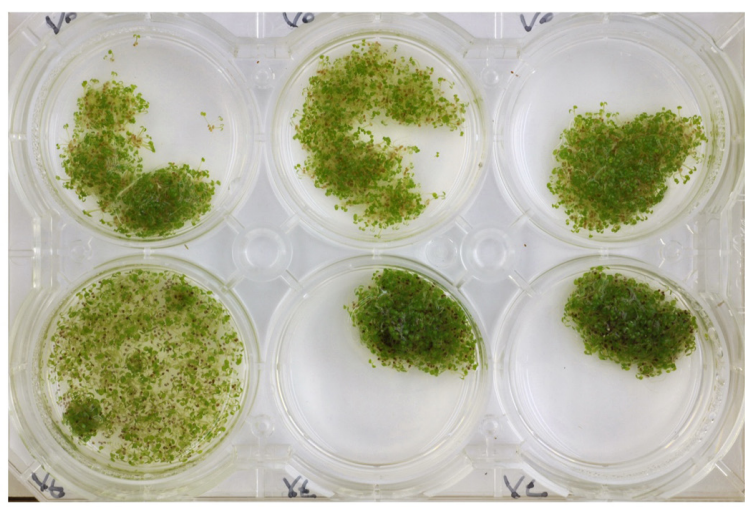

C

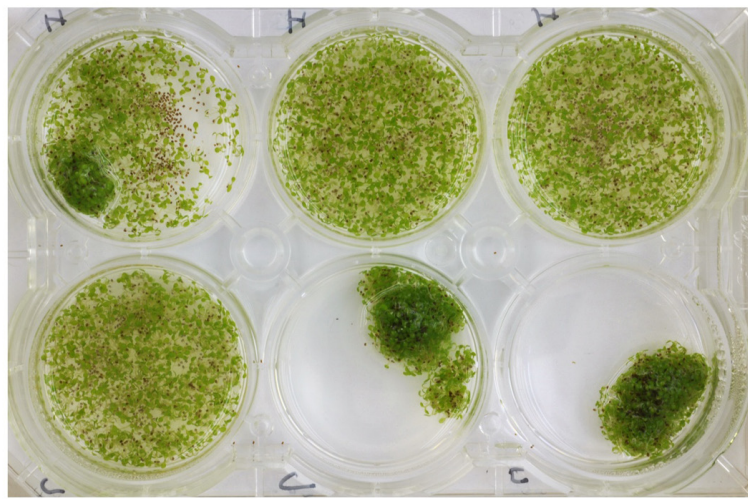

Figure S5 Assay of different mineral waters. Triplicate wells containing $6 \mathrm{~mL}$ of different natural mineral waters (A) Volvic, (B) Vittel, (C) Hepar, and (D) Contrex were inoculated with $20 \mathrm{mg}$ of dry Arabidopsis Col-0 seeds and photographed after 7 days of culture.
EV

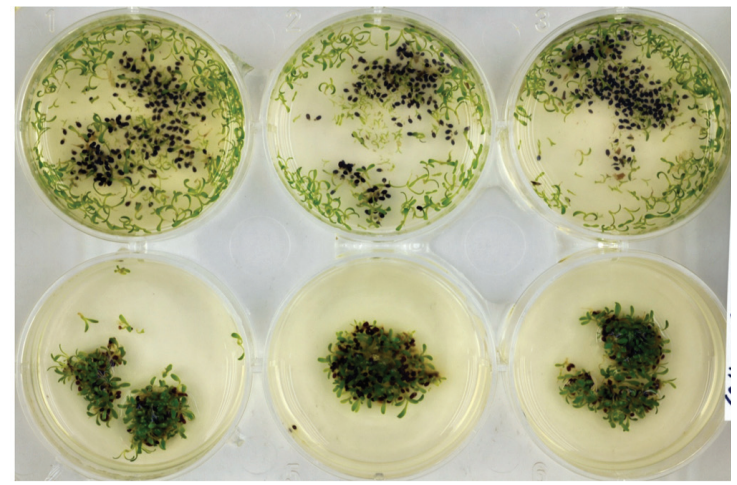

\section{Craterostigma plantagineum}

EV

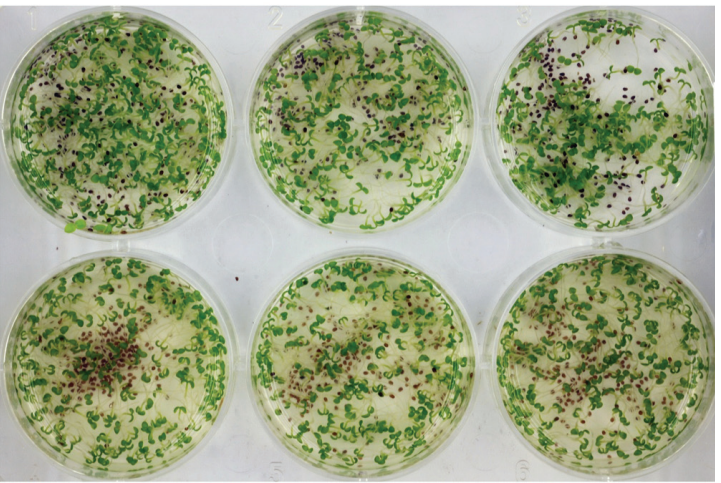

\section{Nicotiana tabacum}

Figure S6 Growth of Craterostigma plantagineum and tobacco seedlings in the system.

Notes: The image shows multiwell plates that were inoculated with seeds of the resurrection plant C. plantagineum or tobacco (Nicotiana tabacum) either in Evian natural mineral water or deionized water, and photographed after 7 days of culture.

Abbreviations: DI, deionized water; EV, Evian natural mineral water.
International Journal of High Throughput Screening

\section{Publish your work in this journal}

International Journal of High Throughput Screening is an international, peer-reviewed, open access journal publishing original research, reports, editorials, reviews and commentaries dedicated to all aspects of high throughput screening, especially related to drug discovery and associated areas of biology and chemistry. The manuscript management sys-

\section{Dovepress}

tem is completely online and includes a very quick and fair peer-review system. Visit http://www.dovepress.com/testimonials.php to read real quotes from published authors. 